\title{
Research on Credit Rating Management of Construction Enterprises Based on Fuzzy Comprehensive Theory
}

\author{
Lichao Jiao ${ }^{1,2}$ \\ ${ }^{1}$ Hebei University of Technology, Tianjin 300401, China \\ ${ }^{2}$ Henan University of Urban Construction, Pingdingshan467044, China
}

Keywords: Construction enterprises, Credit risk, Credit rating, Fuzzy comprehensive theory

\begin{abstract}
Based on the reality of construction business and the basic theory of commercial bank credit ratings, according to the characteristics and actual situation of China's construction enterprises, this paper analyzed the existing rating methodology and theoretical basis, mergingthe fuzzy comprehensive model into enterprises credit ratings, expecting to construct a credit rating index system model for the realities of construction enterprises, providing credible and reliable data for the credit management, forecasting of current credit situation and risk aversion, providing modeling tools for the credit rating and credit risk control of China's construction enterprises, especially small and medium companies and propose corresponding rating management measures.
\end{abstract}

\section{Introduction}

In recent years, the pillar role of China's construction industry in national economy is keep growing, butthe credit deficiency phenomenon is still widespread.The credit rating of Construction Companyhas become an effective means for stakeholder to protect their legitimate rights.The supply of financial market environment is also undergoing profound changes, the decline in the proportion of direct financing became a long-term trend, correspondingly, the financial market emerging various attempts and innovation in the aspect of guarantees, product grade design and credit assessments, etc. Since the inexperienced and imperfections of financial market, therefore, it is urgent to establish a systematic and modeling credit rating for small and medium construction companies.

\section{Analysis on credit rating models}

The author is taking the credit rating system in current Agricultural Bank of China for example to describe the credit rating. The new credit rating system has been implied in Agricultural Bank of China, and the company's credit ratingis mainly financial and non-financial indicators.Detailed in Table 1, in which summarizes various project of construction enterprises' credit rating:

Table 1 main index factors of construction enterprises credit rating

\begin{tabular}{|c|c|c|c|}
\hline \multirow{8}{*}{$\begin{array}{l}\text { Construction } \\
\text { enterprises credit } \\
\text { rating }\end{array}$} & \multirow{4}{*}{$\begin{array}{l}\text { Financial } \\
\text { analysis }\end{array}$} & \multicolumn{2}{|l|}{ Performance evaluation } \\
\hline & & \multicolumn{2}{|l|}{ Solvency evalustion } \\
\hline & & \multicolumn{2}{|l|}{ Profitability evaluation } \\
\hline & & \multicolumn{2}{|l|}{ Management evaluation } \\
\hline & & $\begin{array}{l}\text { Cooperation with } \\
\text { institutions }\end{array}$ & financial \\
\hline & Non-financial & \multicolumn{2}{|l|}{ Leaders quality } \\
\hline & & \multicolumn{2}{|l|}{ Management ability } \\
\hline & & \multicolumn{2}{|l|}{ Forecasting } \\
\hline
\end{tabular}

Above credit index system is a widespread state-owned commercial bank credit rating. The 
financial statements reflect the static history of construction companies, according to its developing strategies, surveying the advantages of the industry to developing new products and other capacities, forecasting dynamic business, which at least analyzing three years to understand the company's development capability and expansion rate. Credit rating index system should establishing credit factors, combining qualitative and quantitative indicators of factors to further ensure the complete index system. We can arrange and put a considerable number of the data and rating of typical construction enterprises and create a model. Stored more data to enrich the model and collection more accurate and timely market information, therefore, we can make more accurate estimate.

\section{Calculation between fuzzy sets}

Assuming A and B are two subsets, the inner product of A and B is the begin membership value, operate with whichever is the lower and get a result, then, the larger value of the final result of the operation, A and B are the two elements to start membership, getting the results of the operation of whichever is the greater, and then take the outer product of the smaller in the operation of the final result. For example:

$$
\begin{aligned}
& A=\frac{1}{t_{1}}+\frac{1}{t_{2}}+\frac{0.85}{t_{3}}+\frac{0.9}{t_{4}}+\frac{0.6}{t_{5}} \\
& B=\frac{1}{t_{1}}+\frac{0.95}{t_{2}}+\frac{0.85}{t_{3}}+\frac{0.8}{t_{4}}+\frac{0.85}{t_{5}}
\end{aligned}
$$

The inner product of $A$ and $B$ is $A_{0} B=(1 \wedge 1) \vee(1 \wedge 0.95) \vee(0.85 \wedge 0.85) \vee(0.9 \wedge 0.8)$ $\vee(0.6 \wedge 0.85)=1 \bigvee 0.95 \vee 0.85 \vee 0.8 \vee 0.6=1$

The outer product of $A$ and $B$ is $A \oplus B=(1 \vee 1) \wedge(1 \vee 0.95) \wedge(0.85 \vee 0.85) \wedge(0.9 \vee 0.8)$ $\wedge(0.6 \vee 0.85)=1 \wedge 1 \wedge 0.85 \wedge 0.9 \wedge 0.85=0.85$

\section{Determination of the membership function value}

In essence, the determination of the membership value of the function should be objective, but in fact it is not a completely objective evaluation criteria process. According to statistics, the index coefficient method is in accordance with the time interval in the proportion of $(0,1)$ to determine the mathematical statistics, establish a "construction company's membership index statistics" to determine the membership function value as an importance reference. The specific determining steps are:First, select 5-7 typical construction companies in the same type which already have the rating, list the name of collection element of each indicator's typical company.Second, determining the comparing element within the same benchmark, select a complex element which have a higher index as reference, its membership function is 1 , then comparing the elements within other indicators, in the closed interval $[0,1]$, the mentioned "construction enterprises evaluation form" is a reference element in specific circumstances of given assessment in accordance with subjective experience, and initially establish a "construction company's contrast ratio fuzzy relation coefficient table”.Third, calculate the close degree among typical construction companies, then sorting be size, taking corresponding relation coefficients $T_{a 1}, T_{a 2}$ and ${ }^{T_{a 3}}$.Fourth, based on the formula,calculate adjustment factor of reach typical companies.Fifth, determine the membership function value of each element and verify the accuracy of each typical construction companies.

In the selected typical construction enterprises, choosing any one regarded as pre-estimate 
company, according to the formula to calculate their credit rating in turn. By calculating to see whether meet the accuracy requirements, and if they does, the calculate method of final membership function is the foundation to estimate the degree of enterprises' evaluation. If not, it should be adjusted and reconsidered until to meet the accuracy requirements.

\section{Calculation method of close degree}

Mapping relation $\sigma \mathrm{F}(\mathrm{U}) \times \mathrm{F}(\mathrm{U}) \rightarrow 0,1 \Rightarrow \sigma \quad(\mathrm{A}, \mathrm{B})$ is the close degree. Fulfill:

(1) $\sigma \quad(\mathrm{A}, \mathrm{B})=1$

(2) $\sigma \quad(\Phi, \mathrm{U})=0$

(3) $\sigma \quad(\mathrm{A}, \mathrm{B})=\sigma \quad(\mathrm{B}, \mathrm{A})$

(4) $\mathrm{A} \subset \mathrm{B} \subset \mathrm{C} \sigma(\mathrm{A}, \mathrm{C}) \leqslant \sigma \quad(\mathrm{A}, \mathrm{B}) \Lambda \sigma \quad(\mathrm{B}, \mathrm{C})$

When estimating construction company credit rating, and calculate the close degree of projects, the key issue is based on the decomposition features of the two companies' key indicators, through reasonable calculation model, to maximize their business to reflect the similarities.

Assuming $\mathrm{N}$ knowing typical construction companies as $\mathrm{A} 1, \mathrm{~A} 2, \mathrm{~A} 3, \ldots,-$, An, its features set $T=\left\{t 1, t 2, \ldots, t_{m}, \mid t_{j}\right.$ as feature element $\}$, feature vector set is $\left\{\mu A i\left(t_{j}\right)|i=1,2,3 \ldots, n j ;| j=1,2,3 \ldots, m\right\}$, then the close degree between $A_{P}$ and $A_{Q}$ is:

$$
\begin{gathered}
\sigma\left(A_{p,} A_{q}\right)=\frac{\sum_{k=1}^{m}\left(\mu_{A p}\left(\mu_{k}\right) \wedge \mu_{A q}\left(\mu_{k}\right)\right)}{\sum_{k=1}^{M}\left(\mu_{A p}\left(\mu_{k}\right) \wedge \mu_{A q}\left(\mu_{k}\right)\right)}(1) \\
(\mathrm{p}, \mathrm{q}=1,2,3 \ldots, \mathrm{n})
\end{gathered}
$$

It can prove that (1) satisfies the close degree axiom system.

In construction company's credit rating, its feature is money (or feature element) decomposition, and comprehensive analysis and determined. Practice shows that the amount of credit rating is different, and the weights effecting on them is $\omega_{1}, \omega_{2} \ldots \omega_{\mathrm{m}}$. and:

$$
\sum_{k=1}^{m} \omega_{k}=1
$$

Then formula (1) can changed into:

$$
\sigma\left(A_{p}, A_{q}\right)=\frac{\sum_{k=1}^{m} \omega_{k}\left(\mu_{A p}\left(\mu_{k}\right) \wedge \mu_{A q}\left(\mu_{k}\right)\right)}{\sum_{k=1}^{M} \omega_{k}\left(\mu_{A p}\left(\mu_{k}\right) \wedge \mu_{A q}\left(\mu_{k}\right)\right)}
$$

In the formula (2), the weight is generally based on the amount of a typical construction company's features, according to the elements to determine its credit rating index. In general, when choosing close formula, the method of Hamming approach or (1) calculates is closer, and Hamming distance reflect the preferably set between the differences within the mode.

Assuming there are two Fuzzy sets A and B, their respect membership function value is $\mu_{A}(x)$ and $\mu_{B}(x)$, the close degree of Hamming is:

Discrete case:

$$
\sigma(A, B)=1-\frac{1}{n} \sum_{k=1}^{m}\left|\mu_{A}\left(\mu_{k}\right)-\mu_{B}\right|(3)
$$

Continuous case: 


$$
\sigma(A, B)=1-\frac{1}{\alpha-\beta} \int_{\beta}^{\alpha}\left|\mu_{A}(X), \mu_{B}(X)\right| d x
$$

(3), (4) represents the average value of the same portion in curves $\mu_{A}(x)$ and $\mu_{B}(x)$.

While using (1) to determine the close degree of set A, B, and when the membership function value is continuous, turning into:

$$
\sigma(A, B)=\frac{\int_{\beta}^{\alpha} \min \left\{\mu_{A}(X), \mu_{B}(X)\right\} d x}{\int_{\beta}^{\alpha} \max \left\{\mu_{A}(X), \mu_{B}(X)\right\} d x}=\frac{k_{1}}{k_{2}}
$$

In the above formula, $k_{1}$ is the same part of the area in $\mu_{A}(x)$ and $\mu_{B}(x)$ two curves, $k_{2}$ is the maximum area surrounded by $\mu_{A}(x)$ and $\mu_{B}(x)$ two curves. Therefore, the equations reflect the same areain set $\mathrm{A}$ and $\mathrm{B}$, and the ratio of $A \cap B$ in $A \cup B$. In order to overcome the "intimate computing”, when comparing two functions, as long as they have the same area, it can use weighted average method to solve, then get formula (6):

$$
\sigma\left(A_{p}, A_{q}\right)=\frac{\sum_{k=1}^{m} \omega_{k}\left(\mu_{A p}\left(\mu_{k}\right) \wedge \mu_{A q}\left(\mu_{k}\right)\right)}{\sum_{k=1}^{M} \omega_{k}\left(\mu_{A p}\left(\mu_{k}\right) \wedge \mu_{A q}\left(\mu_{k}\right)\right)}(6)
$$

Though this calculating method is relatively scientific, we can see that (1) or (2) is correcting Hamming close degree, so in this construction company credit rating, the select of (2) is more close to the base. In the actual calculation, in order to close to the fact and simplicity the calculation, we often use the deformation formula (2), which is uses "closeness" proposed by Professor Wang Peizhuang to calculate, which is:

$$
(A, B)=\frac{1}{2}[A \circ B+(1-A \oplus B)]
$$

Determine of the estimated value of construction company credit rating

Assuming $\mathrm{N}$ typical knowing construction companies, separated as A1, A2 _.--, $\mathrm{Ai}(\mathrm{i}=1,2, \ldots,-., \mathrm{n})$.

$\mathrm{T}$ shows it is using the feature vectors of the degree of companies' credit rating to determine the elementsin this collection, for the broadly describe the effect of construction company's architecture and structural features and fully explain the principle of questions, noted as:

$$
T=\left\{t_{1}, t_{2}, \ldots, \quad t_{j}\right\} \quad(\mathrm{j}=1,2 \ldots \mathrm{m})
$$

The fuzzy subsets of $T$ noted by Zadeh as:

$$
T_{i}=\frac{t_{i 1}}{t_{1}}+\frac{t_{i 2}}{t_{2}}+\ldots+\frac{t_{i j}}{t_{j}}
$$

In the formula, $t_{j}$ is the name of feature elements that effecting construction company credit rating: $T_{i}$ Refers to the corresponding fuzzy subset ofi-th typical construction companies; $t_{i j}$-refers the membership values of $j$-th feature factors effecting credit rating in i-th typical construction companies.

The corresponding fuzzy set of construction companies to be estimated is noted as:

$$
T^{*}=\frac{t_{1}^{*}}{t_{1}}+\frac{t_{2}^{*}}{t_{2}}+\ldots+\frac{t_{j}^{*}}{t_{j}}(8)
$$

$t_{j}^{*}$-refers to the corresponding membership values of $j$-th feature elements, $(j=1,2,3$--- $\mathrm{m}$ )

Supposing the degree of close of a built construction company $\mathrm{Ai}(\mathrm{i}=1,2,3 \ldots, n)$ with the B 
company to be estimated is $\alpha$, and arranged from large to small ordered number of columns $\alpha_{1}, \alpha_{2}, \ldots, \alpha_{n}$, corresponding unit construction company credit rating of $11, A 2 \ldots$, Anis E1, E2, ..., En, based on closeness principle to select three companies with high close degree as the estimation baseline, and to meet:

$\alpha_{1} \geq \alpha_{2} \geq \alpha_{3}$

Then the unit credit ratingestimated value of company $\mathrm{B} \hat{e}_{B}$ is:

$$
\hat{e}_{B}=\lambda\left[\alpha_{1} E_{1}+\left(1-\alpha_{1}\right) \alpha_{2} E_{2}+\left(1-\alpha_{1}\right)\left(1-\alpha_{2}\right) \alpha_{3} E_{3}+\frac{1}{3}\left(1-\alpha_{1}\right)\left(1-\alpha_{2}\right)\left(1-\alpha_{3}\right)\left(E_{1}+E_{2}+E_{3}\right)\right](9)
$$

In (9), $\lambda$ is the adjustment factor, considering the impact of the three selected typical construction companies on the estimate company.

In the prediction formula (9), closeness formula using (1) or (2) to calculate, self-estimation on company A, the result would be equal with the actual construction company credit rating. That is:

$\hat{e}_{A}=e_{A}$

Prove, assuming there are typical similar construction companies A, B, C, and their credit rating respectively is $e_{A}, e_{B}, e_{C}$, their close degree with company A separately is:

$\alpha_{1}=\sigma(A, A), \alpha_{2}=\sigma(A, B), \alpha_{3}=\sigma(A, C)$

Suppose $\alpha_{1} \geq \alpha_{2} \geq \alpha_{3}$, then the predictive value of credit rating is:

$\hat{e}_{A}=\lambda\left[\alpha_{1} e_{A}+\left(1-\alpha_{1}\right) \alpha_{2} e_{B}+\left(1-\alpha_{1}\right)\left(1-\alpha_{2}\right) \alpha_{3} e_{C}+\frac{1}{3}\left(1-\alpha_{1}\right)\left(1-\alpha_{2}\right)\left(1-\alpha_{3}\right)\left(e_{A}+e_{B}+e_{C}\right)\right]$

Select $\lambda=1$, and get:

$$
\hat{e}_{A}=\alpha_{1} e_{A}+\left(1-\alpha_{1}\right) \alpha_{2} e_{B}+\left(1-\alpha_{1}\right)\left(1-\alpha_{2}\right) \alpha_{3} e_{C}+\frac{1}{3}\left(1-\alpha_{1}\right)\left(1-\alpha_{2}\right)\left(1-\alpha_{3}\right)\left(e_{A}+e_{B}+e_{C}\right)
$$

Thanks to $\alpha_{1}=\sigma(A, A)=1$, the above equation two, three and four all is zero.

Rearranging the equation, we can obtain:

$\hat{e}_{A}=e_{A}$

In summary, the prediction model constructed by using close degree (1) and (2) can ensure theprediction value is equal to the actual value, the prediction model is established.

Adjustment the coefficient $\lambda$

In the prediction of Construction Company, we adjust the coefficient $\lambda$ and calculated according to the following empirical formula:

$$
\lambda=1+\frac{1}{m}\left[1.8\left(\frac{T^{\prime}}{T_{a 1}}-1\right)+0.8\left(\frac{T^{\prime}}{T_{a 2}}-1\right)+0.4\left(\frac{T^{\prime}}{T_{a 3}}-1\right)\right](10)
$$

Here $m$ refers to number of elements in construction enterprises fuzzy set.

$T^{\prime}$ With $T_{a 1}, T_{a 2}, T_{a 3}$ represents construction companies to be estimated and the fuzzy relation coefficient of typical construction enterprises with corresponding $a_{1}, a_{2}, a_{3}$.The range of each construction enterprises' fuzzy relation coefficient $T_{i}=\frac{\sum_{j} t_{i j}}{\max \sum_{j=1} t_{i j}}$, is $[0,1](\mathrm{i}=1,2 \ldots, \mathrm{m})$

Construction model is an effective method to ensure scientific assessment, therefore, it is entirely possible to solving the estimation problems in credit rating.Effective use of fuzzy comprehensive evaluation model of credit management system is means that the commercial banks use their own resources and various rating elements to provide services for construction enterprises, especially small and medium enterprises to ease the financial difficulties. We introduced the fuzzy comprehensive theory into the credit rating of construction enterprises, established a corresponding 
model, and explore credit rating management system of construction enterprises. With the help of this platform, it not only helps banks to preliminary screening reputable construction companies, decrease the ratio of bad loan, but also the small and medium enterprises which is real need money to do practice things to find sources of funds.

\section{References}

[1] Li Z. Quantification measure and management analysis on modern credit risk. China Financial Publishing House, Beijing, 2001. (In Chinese)

[2] Yang W. Measure model and management study on commercial bank credit risk. Nanjing University of Aeronautics and Astronautics, Nanjing, 2006. (In Chinese)

[3] Yu Y. Measurement and management of credit risk. Shanghai University of Finance and Economics Press, Shanghai, 2003. (In Chinese)

[4] Zhu S. Innovation and application of enterprises credit rating methodology. Southwestern University of Finance and Economics Press, Chengdu, 2002. (In Chinese)

[5] Basle Committee on Banking Supervision. The internal rating based approach consultative document. Bank for International Settlements, Basle, 2001.

[6] L.C. Thomas. A survey of credit and behavioral scoring: forecasting financial risk of lending to consumers. International Journal of Forecasting, 2000 (16): 149-172. 